\title{
Evolution of Research Reporting - From Excel to QlikView
}

Matthew Schuette, Principal Research Analyst, Enterprise Analytics, University of Kansas Medical Center

I

$\mathrm{n}$ the last ten years, the University of Kansas Medical Center (KUMC) has experienced strong growth in its academic, clinical and research enterprises. From 2004 to 2012 , the basic sciences grew by $15 \%$ in total full-time faculty and post-doctoral students, while the number of faculty PIs and post-doctoral students within clinical departments rose by over $40 \%$. Consequentially, total research expenditures increased by over $50 \%$ during this time, with some additional boosting by federal stimulus funds. As the enterprise has grown, the number of research centers has expanded. Frontiers was created in 2011 after KUMC received a Clinical and Translational Research Award from the NIH. In 2012, the KU Cancer Center achieved National Cancer Center Institute designation, a top priority of the Medical Center since 2005.

The importance of accessible data, high-quality reporting, and analytics for both research and financials escalated during this time, shaped by enterprise growth and leadership focus. With the advent of sophisticated business intelligence tools, the time was ripe for the Medical Center to evolve its reporting environment: From Excel to QlikView.

\section{Enterprise Analytics}

The lead partner in business intelligence (BI) and institutional research (IR) at KUMC is the Office of Enterprise Analytics (EA). Until very recently, the office was housed under Academic Affairs and was called the Office of Planning \& Analysis. The name change occurred in 2011 to reflect the broadened scope of the department, as well as its movement to report under Administration. The department is led by Dr. Russ Waitman, who also serves as Director of Medical Informatics at KUMC. The current EA team has enhanced skills in data mining, analysis, and reporting with backgrounds stemming from academics, data management, finance and accounting, and application development.

Up until about 2004, EA primarily served the university with student-centered IR functions, and the makeup of the team was significantly different. The main responsibilities involved academic affairs support including compliance reporting to the Kansas Board of Regents, federally-mandated IPEDS submissions, coordinating and/or completing external surveys pertinent to KUMC programs, and ad-hoc reporting on student and faculty data. At that time, EA did not have the necessary knowledgebase or impetus to support the research enterprise. In 2006, there was a concentrated focus to develop a framework that would allow for effective and timely research reporting using the programming and data 
skills within the Enterprise Analytics team. These efforts led to a surge in requests from the research Vice Chancellor, KUMC's Research Institute, and department, center, and grant administrators. Further integration with financial data allowed for grant expenditure reporting.

Starting in 2009 the institution began looking heavily into comprehensive financial tracking and an appropriate $\mathrm{BI}$ tool for this venture. This initiative led to hiring RSM McGladrey to introduce strategies for organizing the underlying data and security structures along with the initial development of the main QlikView application. [Note: QlikView is the BI product of QlikTech, Inc. and is described below.] The office continues to be molded by the changing dynamics and needs of the Medical Center, as well as the trend institutionally toward BI and self-service reporting functionality.

The Data and the Tools
The primary source of research and financial data is PeopleSoft (PS) Enterprise Financial, Grants, and Human Capital Management systems. One of the vital roles of Enterprise Analytics is to mine, massage, and join tables from PS, and to use internal business practice rules to create consolidated tables for either direct reporting needs or to supply the backbone for an online BI reporting environment (e.g. QlikView, Tableau, SAS Enterprise BI). Prior to the implementation of QlikView (QV) on campus, most research data tables and reports were created on-demand using SAS data steps, procedures, and SQL queries. The use of SAS as a data mining and consolidation tool remains high, specifically for ad-hoc reporting and areas where development in a BI tool would not be cost- or time-effective.

Up until the BI-era at KUMC, nearly all research reports were delivered with

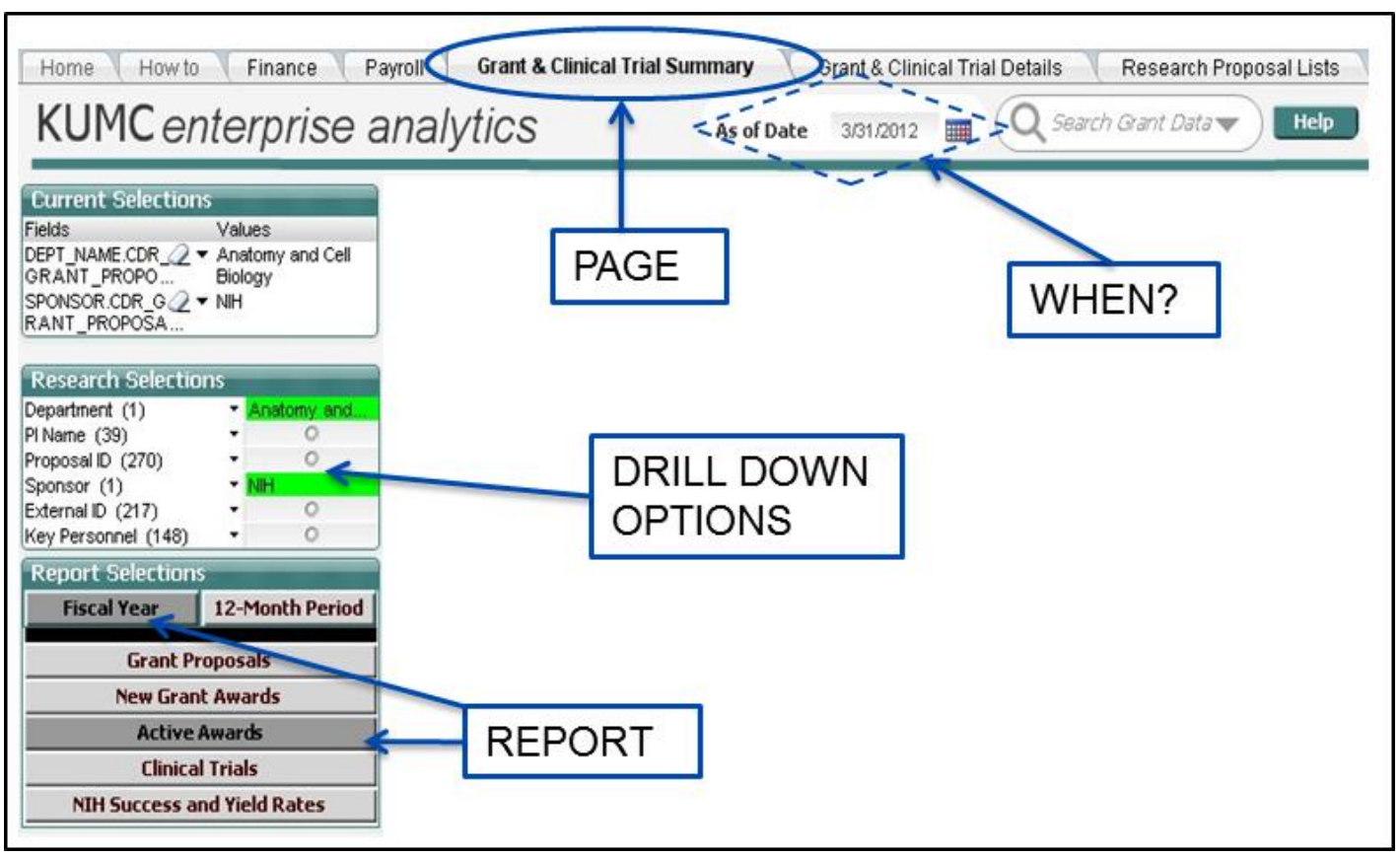

Figure 1: Typical layout of a QlikView page in EA's main application 
Excel. SAS provides easy exporting and importing of Excel files, and most staff on campus are familiar with its features. The use of Excel for ad-hoc reporting will continue for the foreseeable future. BI tools require moderate training in the use of developed applications, as well as security access being granted.

QlikView is a business intelligence tool which is highly flexible, has a rich, visual user interface, and allows users to clearly see associations between data. Because the engine behind associative searching is in-memory, no queries are fired when a user clicks on a data point. This allows for nearly real-time analysis, as any dashboards are quick to regenerate when selections are made. KUMC currently uses Microsoft SQL Server Integration Services (SSIS) to create warehoused tables from source systems. Further ETL occurs to provide QlikView with intermediate files (called QVDs) for faster access to data (as opposed to hitting the source systems). Additional scripting may be done within a QlikView application (QVWs) to produce the polished objects within the application.

\section{Standard Research Reporting}

During the presentation, I outlined four of the standard research reports which Enterprise Analytics provides. Additionally, the office assists KUMC's Research Institute with annual reporting and there are many ad-hoc requests that we receive each year. With the emergence of QlikView and subsequent training of users, the number of ad-hocs has lessened to some degree. I will discuss the basics behind each report and, where appropriate, the evolution of that report in QlikView.

\section{Monthly Reports}

The monthly reports were created to provide administrators with an overall look at grant and clinical trial activity at the end of each month, while showing year-to-year trends. The raw data were produced with SAS, exported to Excel templates, and then further formatted. The pages of each report showed tables and charts of full fiscal year, fiscal year-

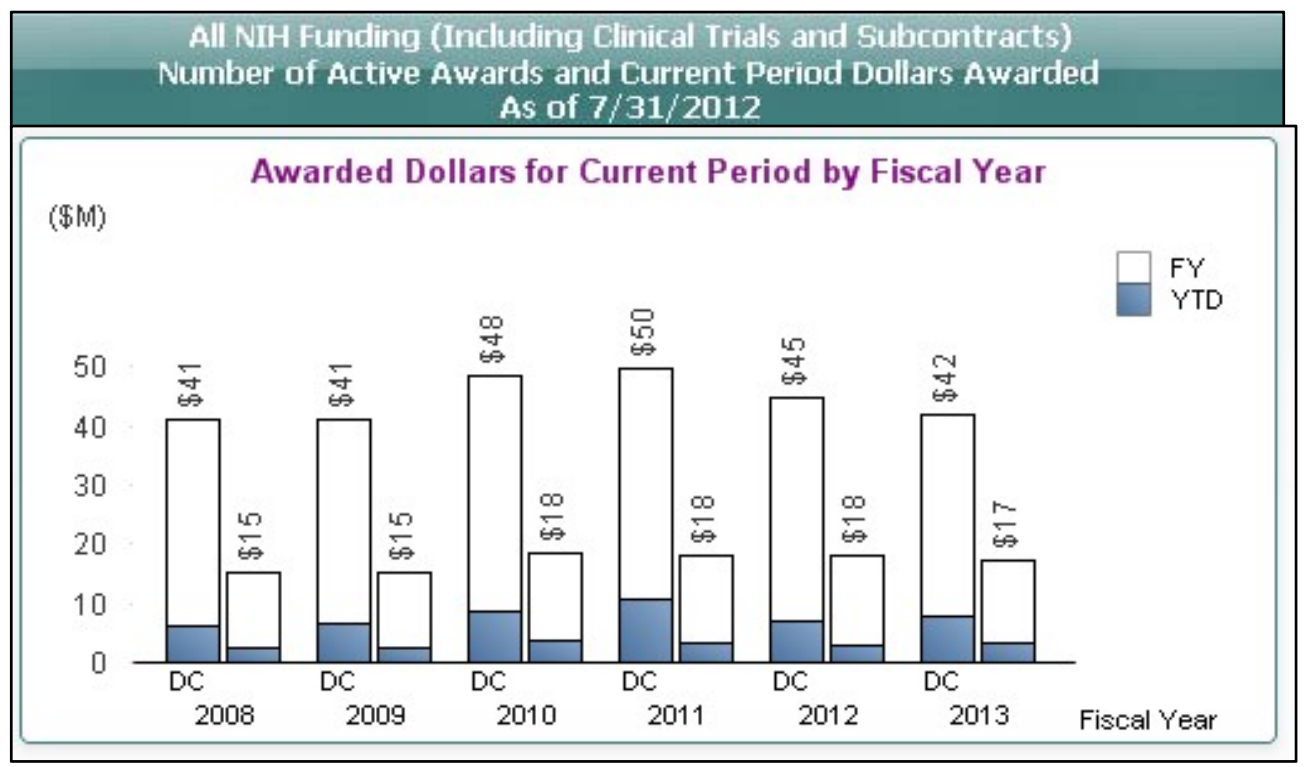

Figure 2: A typical report/chart from the research details page in QlikView 
to-date, and 12-month period. Top administrators were also provided lists of new submissions, new awards, and the status of proposals. An analyst would

\section{NIH Funding Ranks}

Prior to 2006, the NIH used to publish rankings of NIH awards to medical schools, based on total dollars awarded

\begin{tabular}{|l|l|l|r|r|r|r|}
\hline \multicolumn{2}{|c|}{ University of Kansas Medical Center - Kansas City and Wichita Campuses } \\
Current Efforts
\end{tabular}

Figure 3: A small portion of the efforts report in QlikView

typically spend 12-20 hours per month compiling and fine-tuning each report. In $\mathrm{QV}$, the underlying grant tables were created as QVDs and the Excel reports were mimicked. QV allows the user to tailor the report to a finer level (e.g. selecting a single department) and to use "as-of" dating.

\section{Investigator Percent Effort and NIH Other Support}

Another piece in the development of research tables was to provide KUMC's Research Institute (RI) with quickly-delivered reports on investigator percent effort as well as formatted NIH Other Support documentation. Currently, these are in the form of Excel tables and Word files, and EA receives 300-400 of these requests per year. While the process is almost entirely automated from a data-consolidation perspective, it requires about 2-3 hours per week of an analyst's time to format the reports. QlikView development of these same reports is in finishing stages, so that time will be freed up on the EA side, and the convenience is provided to the RI and other department administrators to get the information whenever they need it. during the federal fiscal year. When the NIH online portfolio came on-board, such rankings were no longer produced. For internal purposes, KUMC and Enterprise Analytics began to produce NIH rankings, both overall and at the department level, and to disaggregate between public schools of medicine and all schools of medicine (which NIH did not do). The $\mathrm{NIH}$ RePORT tool allows anyone to download NIH award data or to perform refined searches. Historically, EA provided rankings to KUMC research or department administrators, and also produced summary reports for our website. From a national standpoint, the Blue Ridge Institute for Medical Research stepped in and filled the role of producing and publicizing rankings (no public school breakout). In QlikView, the NIH Rankings report is available to all users, and provides both yearly detail and trending information. The advantage with QV is that the user can select any institution/school/department, one or multiple years, and to view public or overall rankings. 


\begin{tabular}{|c|c|c|c|}
\hline & $\begin{array}{l}\text { NIH Funding Ranks by Department } \\
\text { Public Schools of Medicine } \\
2012 \text { *All Departments }(\mathrm{N}=83 \text { ) }\end{array}$ & & 回XI国- 듬 \\
\hline Rank $>$ & School & Awards & Funding \\
\hline 31 & University of Texas Medical School at Houston & 155 & $\$ 61,539,389$ \\
\hline 32 & Wayne State University School of Medicine & 151 & $\$ 51,796,319$ \\
\hline 33 & University of Arizona College of Medicine & 120 & $\$ 51,612,190$ \\
\hline 34 & University of Kansas School of Medicine & 129 & $\$ 49,843,792$ \\
\hline 35 & University of Nebraska College of Medicine & 119 & $\$ 47,780,523$ \\
\hline 36 & University of Connecticut School of Medicine & 113 & $\$ 43,824,070$ \\
\hline
\end{tabular}

Figure 4: A report listing public schools of medicine NIH funding in QlikView

\section{Departmental "Scorecards"}

These reports are delivered to the Vice Chancellor of Research and provide a complete fiscal year listing of projects by department as well as information on paid effort vs. committed effort for individual faculty in the School of Medicine. All reports are Excel formatted. There is no intention to integrate these reports into the QlikView environment.

\section{Final Thoughts}

Although the title of the presentation invoked thoughts of complete transition, it should be noted that both Excel and QlikView are used in conjunction. For example, source-transformed tables, cre ated in SSIS or QV, can be mined and analyzed with SAS, which often is better suited for frequency analysis and other types of data validation. Also, users of QV are trained to export tables to Excel to do fine-tuning or further analysis. In conclusion, the advent of BI tools, quicker and relatively cheaper computing memory and power, and enhanced institutional focus, has led KUMC into a newer world of data mining, intelligent and self-service reporting, along with data and analytically-driven decision making. The Enterprise Analytics team is central to these ongoing efforts. 